\title{
Correspondence
}

\section{Approximating Performance Measures for Public Services}

Oded Berman and Sandeep Vasudeva

\begin{abstract}
This paper deals with estimating performance measures, such as average response time for spatially distributed networks. Demands are generated stochastically at the nodes and the service units are stationed at service centers when available. Whenever a call arrives, a service unit will travel to the call's location. When there are no available servers, the call will enter an infinite capacity queue at that node. The service units will travel from node to node serving the calls and return to the service center only when there are no more calls waiting. In most cases, exact models are too complicated to analyze. This paper presents approximations which are tested using simulation and found to give good results.
\end{abstract}

Index Terms-Hypercube model, location, minimizing response time, multifacility, multiserver, queueing.

\section{INTRODUCTION}

This paper deals with the problem of estimating performance measures for spatially distributed networks of demand nodes serviced by mobile servers. These types of networks are frequently encountered in public sector service industries. The demand nodes can be thought of as representing city neighborhoods connected by roads. Each node represents demands for service originating from that neighborhood. The demands are generated stochastically and the service units, stationed in service centers, go and service these calls according to a preselected policy.

While considerable literature exists on modeling a network of demand nodes, most of it assumes that every demand has to be serviced from the service center, i.e., the service unit goes back to the service center after every service. This assumption is quite limiting for many services. The model presented in this paper assumes that the service unit goes back to the service center whenever free but travels from node to node to service the calls as long as there are calls waiting in the queue. This assumption is suitable to a variety of services. For example, in the case of police, if after completing the service, the response unit finds no other call waiting in the queue, it comes back to the service center. However, if there are other calls waiting in the queue, the response unit may be dispatched to the scene of the next waiting call directly from the scene of the previous call. In case of services such as telephone, electricity, and consumer gas supply, maintenance of water supply and sewage disposal systems, the service unit does not require to go back to the service center after every service unless replenishment of stocks is due.

In these type of service environments, the performance measure of interest is very often the average response time to a random call for service. However, an exact estimation of the average response time in the general case is very difficult as the service times are not independent identically distributed.

Manuscript received November 27, 2000; revised September 20, 2003, September 30, 2003, June 16, 2004, and November 2, 2004. This paper was supported by a grant from NSERC. This paper was recommended by Associate Editor Y. Narahari.

The authors are with the Joseph L. Rotman School of Management, University of Toronto, Toronto, ON M5S 3E6, Canada (e-mail: berman@ rotman.utoronto.ca).

Digital Object Identifier 10.1109/TSMCA.2005.850607
Larson [1] formulated the problem as a hypercube queueing model to obtain various performance measures for a network of practical size. The model assumes that calls arrive according to a Poisson process and service times are exponentially distributed. Given the home locations of $N$ service units and fixed dispatch rules, the model solves $2^{N}$ linear simultaneous equations. In [2], Larson proposed an approximate method to solve the problem with $N$ nonlinear simultaneous equations, thus making the model useful for larger-sized problems. By introducing a normalizing technique known as mean service time calibration [3], the hypercube model can include travel times as a part of the total service time.

In [4], Berman et al. analyzed a network of demand nodes with a single mobile service unit operating from a service center. Each node generates demands according to a Poisson process. The travel times between the service center and the node are considered explicitly as parts of the total service time. The service policy is first come first serve (FCFS) and it was assumed that the service unit must return to the service center after every service which allowed the system to be modeled as an $M / G / 1$ queue. The algorithm for finding the optimal location was made more efficient for the special case of a tree network by the authors in [5] which exploits the convexity properties of the objective function on paths of the tree and in [6] and [7], Chiu analyzed the trajectory of the Stochastic Queue Median in general and in the special case of a tree network. In [8], Batta developed an efficient algorithm to find the optimal facility location on a network, parametrically in the arrival rate of calls, when the choice of location is limited to a finite set of discrete points.

In [10], the $M / G / 1$ queueing system of the original 1-server model was extended by Batta and Berman to an $M / G / k$ queueing system for a single facility that houses $k$ servers. Using Nozaki and Ross's approximation for the expected waiting time in an $M / G / k$ queue, several useful results were derived for optimal location of a multiserver facility. Berman et al. [11] derived heuristic procedures for locating $p$ mobile servers on a network in the presence of queueing-like congestion.

For a comprehensive review of the literature on the above problem and other extensions, the reader can refer to [13] and [15].

In [12], Jamil et al. discussed the problem of finding the optimal home location of a single service unit in a network operating under the service policy of going from call to call so long as the queue of incoming calls is not empty and coming back to the service center only when there is no call waiting in the queue. The analysis was performed for the FCFS service discipline via a busy period analysis in the case of a limited capacity queue. The authors showed that the optimal home location as well as the average response time were significantly different from those obtained in [4].

While the papers [1]-[11] above use the assumption that the server return to the service center after every service, the model presented in [12], being mathematically complex, could not be extended to the practical case of multiserver multifacility location problem. This is the main reason for developing an approximate model for the problem that could generate a simple closed form expression for the waiting time that can also be incorporated into the multiserver multifacility location problem. 


\section{Approximating Average Response TIME For AN EMERgENCY SERVICE With Single SERVICE FACILITY}

We now present the approximation model for the average response time. The following assumptions are made.

1) Each node of the network generates Poisson distributed demands from an infinite population.

2) There is a single server moving (with unit velocity) on the network to service demands originating at different nodes. All the calls enter a single queue and are serviced according to the FCFS discipline.

3) The unit returns to the service center only if there are no calls waiting in the queue. Then, the next call that arrives has to be serviced from the service center even if the call arrives while the service unit is returning to the service center.

In the approximation model developed below, we calculate the first and second moments of the service times, defined as the sum of travel time to reach the location of the demand and the on-site service time, and assume that they are independent identically distributed even though they are not.

$\lambda \quad$ Total demand arrival rate.

$h_{i} \quad$ Fraction of demand originating at Node $i\left(\sum_{i} h_{i}=1\right)$.

$R_{i} \quad$ Constant on-site service time required for a demand from Node $i$.

$\rho \quad$ Fraction of demands that find the queue nonempty.

$l_{i j}=l_{j i} \quad$ Distance between Nodes $i$ and $j$.

$r \quad$ Constant time required to be spent (e.g., for replenishment) once the server returns to the service center.

$d(x, i) \quad$ Distance from the service center (denoted by $x$ ) to Node $i$.

Note that in our approximation model, the server is considered free if she has finished serving the calls assigned to her and the queue in front of her is empty. The server may be at the service center or travelling toward the service center. The server is busy as soon as a new call is assigned to her and continues to be busy as long as all the calls that have queued up before her do not get serviced.

Consider a call that initiates a busy period. This call may occur while the server is available at the service center or while the service unit is travelling back to the service center after servicing all waiting calls. Let $\tilde{d}(i, x, r)$ be the random variable denoting time duration since a call initiating a busy period occurs till the server starts travelling toward that call from the service center given that the last call served is at Node $i$. Let $d^{\prime}(i, x, r)$ be the expected value of this random variable. It is easy to verify that

$$
d^{\prime}(i, x, r)=[d(x, i)+r]-\frac{1}{\lambda}[1-\exp (-\lambda(d(x, i)+r))]
$$

Obviously, when $\lambda$ is large, $d^{\prime}(i, x, r) \rightarrow[d(x, i)+r]-(1 / \lambda)$ and when $\lambda \rightarrow 0, d^{\prime}(i, x, r) \rightarrow 0$.

\section{A. Calculating the Average Response Time}

One of the key findings of this paper is that the service times can be assumed to be independent identically distributed, even when the server does not go back to the service center after every service. As shown later, the models based on this assumption give reasonably good computational results.

With this assumption, the system behaves like a modified $M / G / 1$ queue with a different service-time distribution for calls that arrive when the server is free (calls that initiate a busy period) and for calls that arrive during the busy period.

Then, from [14], we obtain the following expression for the average queueing delay:

$$
W_{q}=\left\{\begin{array}{cc}
{\left[\frac{\lambda}{1-\lambda(K-S(x))}\right]\left[\frac{A}{2(1-\lambda K)}\right],} & \text { if } 1-\lambda K>0 \\
+\infty, & \text { otherwise }
\end{array}\right\}
$$

where

$$
\begin{aligned}
A=\sigma_{1}^{2}(x)+(S(x))^{2}+\lambda[S(x) & \left\{\sigma_{2}^{2}+K^{2}\right\} \\
& \left.-K\left\{\sigma_{1}^{2}(x)+(S(x))^{2}\right\}\right] .
\end{aligned}
$$

$\sigma_{1}^{2}(x)$ and $\sigma_{2}^{2}$ represent the variance of the service time for calls initiating a busy period and for subsequent calls in a busy period, respectively, $S(x)$ is the overall average service time given the server is free when a call is placed and $K$ is the overall average service time given the server is busy when a call arrives. We acknowledge that when using the modified $M / G / 1$, we ignore the fact that the distribution of the first service time is dependent on the last customer served in the previous busy period. As noted earlier our models give reasonably good computational results.

1) Calculating $\rho$ and the Average Service Time: In order to calculate the average service time for the network, we need to calculate the average service time for demands originating at each node separately and then take a weighted average as shown below.

When the server is busy at a node at the time a call from Node $i$ is placed, the average distance travelled to service the call is $\sum_{j} h_{j} l_{j i}$.

Thus, the average service time for the call is

$$
L_{i 2}=\sum_{j} h_{j} l_{j i}+R_{i}
$$

When the server is free at the time a call from Node $i$ is placed, the average service time for the call is

$$
L_{i 1}=\sum_{j} h_{j} d^{\prime}(j, x, r)+d(x, i)+R_{i}
$$

Therefore, the average service time for the call from Node $i$ is

$$
L_{i}=(1-\rho) L_{i 1}+\rho L_{i 2} .
$$

By definition of $\rho$ as the fraction of time a demand finds the server busy

$$
\rho=\lambda \sum_{j} h_{j} L_{j}
$$

From (5) and (6), $\rho=\lambda \sum_{j} h_{j}\left[(1-\rho) L_{j 1}+\rho L_{j 2}\right]$.

Using (3) and (4)

$$
\rho=\lambda[(1-\rho) S(x)+\rho K]
$$

where $S(x)$ is the overall average service time given the server is free when a call is placed

$$
S(x)=\sum_{k} h_{k} d^{\prime}(k, x, r)+\sum_{j} h_{j} d(x, j)+\sum_{j} h_{j} R_{j}
$$

and $K$ is the overall average service time given the server is busy when a call arrives

$$
K=\sum_{j} h_{j} \sum_{k} h_{k} l_{k j}+\sum_{j} h_{j} R_{j}
$$


From (7), we get

$$
\rho=\frac{\lambda S(x)}{1+\lambda S(x)-\lambda K}
$$

For stability of the queueing system, we must have $\rho<1$, which implies [from (10)] that $\lambda K<1$. No matter how big the queue size is, given $\lambda K<1$, the queue will be depleted sooner or later and therefore the probability of a demand finding the server busy will be less than one. We note that our condition for stability is only a necessary condition.

2) Calculating the Second Moment of the Service Times: The second moment is required for calculating the waiting times according to (1) and (2). Let $S^{2}(x)$ represent the second moment of the overall service time. Then

$$
S^{2}(x)=\sum_{j} h_{j} L_{j}^{2}
$$

where $L_{j}^{2}$ is the second moment of the service time for a call arriving at Node $j$ and is equal to

$$
L_{j}^{2}=(1-\rho) L_{j 1}^{2}+\rho L_{j 2}^{2}
$$

$L_{j 1}^{2}$ and $L_{j 2}^{2}$ are, respectively, the second moments of the service times given that the calls arrive when the server is free and busy. Then, from (3)

$$
L_{j 2}^{2}=\sum_{k} h_{k}\left(l_{k j}\right)^{2}+R_{j}^{2}+2 R_{j} \sum_{k} h_{k} l_{k j}
$$

and from (4), denoting $\sum_{k} h_{k} d^{\prime}(k, x, r)$ by $Q(x)$

$$
\begin{aligned}
L_{j 1}^{2}=\sum_{k} h_{k} E\left[\tilde{d}(k, x, r)^{2}\right]+d(x, j)^{2}+R_{j}^{2} & \\
& +2 R_{j} Q(x)+2 R_{j} d(x, j)+2 d(x, j) Q(x) .
\end{aligned}
$$

Hence, from (11) to (14)

$$
\begin{aligned}
S^{2}(x)=(1-\rho) \sum_{j} h_{\curlywedge}[ & E\left[\tilde{d}(j, x, r)^{2}\right] \\
& +d(x, j)^{2}+R_{j}^{2}+2 R_{j} Q(x)+2 R_{j} d(x, j) \\
& +2 d(x, j) Q(x)]+\rho \sum_{j} h_{j} L_{j 2}^{2} .
\end{aligned}
$$

Also, it is easy to show

$$
\begin{aligned}
E\left[\tilde{d}(k, x, r)^{2}\right]=(d(x, k) & +r)^{2}-\frac{2(d(x, k)+r)}{\lambda} \\
& +\frac{2}{\lambda^{2}}(1-\exp (-\lambda(d(x, k)+r))) .
\end{aligned}
$$

Hence, the second moment of the overall service time is known. Since (2) can be rewritten as

$$
A=\sum_{j} h_{j} L_{j 1}^{2}+\lambda\left[S(x)\left\{\sum_{j} h_{j} L_{j 2}^{2}\right\}-K\left\{\sum_{j} h_{j} L_{j 1}^{2}\right\}\right]
$$

from (1), (10), (11), and (12), we get the average waiting time in queue for our case as

$$
\begin{aligned}
W_{q}= & \frac{\lambda S^{2}(x)}{2(1-\lambda K)} \\
= & \frac{\lambda \rho \sum_{j} h_{j} L_{j 2}^{2}}{2(1-\lambda K)}+\frac{\lambda}{2}\left(\frac{1}{1+\lambda S(x)-\lambda K}\right) \\
& \quad \times \sum_{j} h_{j}\left[E\left[\tilde{d}(j, x, r)^{2}\right]+d(x, j)^{2}+R_{j}^{2}\right. \\
& \left.\quad+2 R_{j} Q(x)+2 R_{j} d(x, j)+2 d(x, j) Q(x)\right] .
\end{aligned}
$$

TABLE I

COMPARISON OF AVERAGE RESPONSE TIMES FOR EXAMPLE 1

\begin{tabular}{c|c|c|r}
\hline$\lambda$ & Model Average Response Time & Simulation Average Response Time & \% Error \\
\hline 0.1 & 0.625 & 0.626 & -0.2 \\
\hline 0.2 & 0.689 & 0.692 & -0.4 \\
\hline 0.3 & 0.762 & 0.764 & -0.3 \\
\hline 0.4 & 0.845 & 0.849 & -0.5 \\
\hline 0.5 & 0.944 & 0.946 & -0.2 \\
\hline 0.6 & 1.063 & 1.065 & -0.2 \\
\hline 0.7 & 1.212 & 1.212 & 0 \\
\hline 0.8 & 1.408 & 1.405 & 0.2 \\
\hline
\end{tabular}

Further, using (6), the expected response time which includes also a travel time component is

$$
T(x)=W_{q}+\sum_{j} h_{j}\left(L_{j}-R_{j}\right)=W_{q}+\frac{\rho}{\lambda}-\sum_{j} h_{j} R_{j}
$$

which, using (10), can be written as

$$
\begin{aligned}
T(x)= & \frac{\lambda^{2}}{2}\left(\frac{S(x)}{(1-\lambda K)(1+\lambda S(x)-\lambda K)}\right) \\
& \times \sum_{j} h_{j} L_{j 2}^{2}+\frac{\lambda}{2}\left(\frac{1}{1+\lambda S(x)-\lambda K}\right) \\
& \times \sum_{j} h_{j}\left[E\left[\tilde{d}(j, x, r)^{2}\right]+d(x, j)^{2}+R_{j}^{2}+2 R_{j} Q(x)\right. \\
& \left.\quad+2 R_{j} d(x, j)+2 d(x, j) Q(x)\right] \\
& +\frac{S(x)}{1+\lambda S(x)-\lambda K}-\sum_{j} h_{j} R_{j} .
\end{aligned}
$$

For brevity, we write it as

$$
\begin{aligned}
T(x)= & \frac{\lambda^{2}}{2}\left(\frac{S(x)}{(1-\lambda K)(1+\lambda S(x)-\lambda K)}\right) M \\
& +\frac{\lambda}{2}\left(\frac{1}{1+\lambda S(x)-\lambda K}\right) N(x) \\
& +\left(\frac{S(x)}{1+\lambda S(x)-\lambda K}-R\right)
\end{aligned}
$$

where $M$ and $N(x)$, respectively, are the second moments of the service time given the server is busy and free.

Example 1: In Table I, we compare the values of the average response time for various $\lambda$ values given by the approximate model presented above with those obtained from simulation for the 5-node network with parameters

$l_{12}=3, l_{13}=3, l_{14}=11, l_{15}=9, l_{23}=5, l_{24}=8, l_{25}=$ $11, l_{34}=13, l_{35}=6, l_{45}=7 ; h_{1}=h_{2}=h_{5}=0.15, h_{3}=$ $h_{4}=0.275$; server's travel speed $=10, R_{i}=0.1$ (constant) for $i=$ $1, \ldots, 5$. The service center is at Node 2 .

As can be seen from Table I, the errors are very small (less than $0.5 \%)$.

\section{B. Trajectory of the Optimal Location}

It can be seen from (19) that when $\lambda \rightarrow 0$, the third term dominates and therefore

$$
\begin{aligned}
T(x) & \simeq S(x)-\sum_{j} h_{j} R_{j} \\
& =\sum_{k} h_{k} d^{\prime}(k, x, r)+\sum_{j} h_{j} d(x, j) .
\end{aligned}
$$

Therefore, when $\lambda$ is very small, the average response time is minimized when the service center is located at the point that minimizes (20) which we call the 'modified median'. When $\lambda$ increases, the second 
term becomes important and the optimal location reflects a trade off between $S(x)$ and $N(x)$, i.e., the optimal location will be somewhere on the path joining minimum $S(x)$ location with minimum $N(x)$ location.

We can combine terms and rewrite (19) as

$$
\begin{aligned}
T(x)=\frac{\lambda}{2(1-\lambda K)}+\left(\frac{1}{\lambda}\right)\left(\frac{1}{1+\lambda S(x)-\lambda K}\right) \\
\quad \times\left(\frac{\lambda^{2}}{2}(N(x)-M)-(1-\lambda K)\right)+\frac{1}{\lambda}-R .
\end{aligned}
$$

From this equation, we can get the following insights.

1) For $N(x)<M, T(x)$ increases with the increase of both $N(x)$ and $S(x)$ and, hence, the optimal location is that provides the best tradeoff between $N(x)$ and $S(x)$.

2) For $N(x)>M$ and $\left(\left(\lambda^{2} / 2\right)(N(x)-M)-(1-\lambda K)\right)<$ $0, T(x)$ again increases with the increase of both $N(x)$ and $S(x)$ and the optimal location once again represents the best trade off between the two depending upon $\lambda$. These conditions hold for $\lambda \in\left(0, \lambda^{\#}\right)$ where

$$
\lambda^{\#}=\frac{-K+\sqrt{K^{2}+2(N(x)-M)}}{(N(x)-M)}<\frac{1}{K} .
$$

3) For $N(x)>M$ and $\left(\left(\lambda^{2} / 2\right)(N(x)-M)-(1-\lambda K)\right)>$ $0, T(x)$ increases with the increase in $N(x)$ but decreases with the increase in $S(x)$ and therefore the optimal location moves away from the modified median toward minimum $N(x)$ location. This happens for $\lambda \in\left(\lambda^{\#},(1 / K)\right)$.

\section{Optimal Location Versus Optimal Policy}

When $\lambda$ is small, the service center is located near the modified median. Therefore we would expect $S(x)<K$ and $N(x)<M$. For some special types of networks, e.g., a star network with the service center located at the center, this condition will hold for all $\lambda \in$ $(0,(1 / K))$ and having a service center is always better than travelling from node to node. However, in general, when $\lambda$ increases, $S(x)$ and $N(x)$ increase, while $M$ and $K$ remain the same and therefore for large $\lambda^{\prime}$ s, policy of travelling from node to node gives a lower average response time. However, a trip to the service center with a certain frequency is desirable for many types of services. Therefore, if a policy with a service center gives a lower average response time, it dominates the policy of travelling from node to node but the converse is not true. If a policy with a service center gives a higher average response time, it might still lie on the efficient frontier of a response time-visiting frequency graph.

Interestingly, in the case of a service policy of going to the service center when free, for $N(x)>M, \lambda \in\left(\lambda^{\#},(1 / K)\right)$, the queueing is not well behaved as $T(x)$ decreases with the increase in $S(x)$, other parameters remaining the same. This can lead to the failure of the policy of going back to the service center whenever the server is free to lie on the efficient frontier discussed above in some cases. In other words, it may be possible to find another policy that uses the same frequency of visiting the service center but gives a lower average response time.

\section{Multiserver One-Station NeTWORKS}

We now generalize the model developed earlier to the case when there is one service center on the network that houses a number of servers.

\section{A. Approximating $W_{q}$}

We use the approximate expression by Nozaki and Ross [10] for calculating the queueing delay for the $M / G / \mathrm{P}$ system seen in (22a) at the bottom of the page, where $S(x)$ and $S^{2}(x)$ are the first and second moments of the service time. However, in addition to having different service-time distributions for calls arriving in the free and busy periods, now we also have different effective arrival rates $\lambda_{1}$ and $\lambda_{2}$ when a server is free and busy, respectively (as explained in Section III-B). Then, from [9], we rewrite $W_{P}(x)$ as can be seen in (22b) at the bottom of the page, where

$$
S^{2}(x)=(1-\rho) \sum_{j} h_{j} L_{j 1}^{2}+\rho \sum_{j} h_{j} L_{j 2}^{2}
$$

and the fraction of time a call finds all the servers busy, $\rho$ is

$$
\rho=\frac{\lambda_{2} S(x)}{1-\lambda_{2}(K-S(x))}
$$

whereas, for computing $S(x)$ and $S^{2}(x)$, we require $\lambda_{1}$. This is explained in Section III-C.

\section{B. Calculating $\rho$ for Multiple Servers}

First, consider a network with $P=2$. We approximate this system by considering two independent $M / G / 1$ queues, one for each server with an arrival rate of $(\lambda / 2)$ for each. Let $\rho^{*}$ be the fraction of time a call finds its respective server busy. Then, given that one server is free and the other one busy, an incoming call to the busy server will be diverted to the free server. Therefore, when a server is free, the effective arrival rate $\lambda_{1}$ is

$$
\lambda_{1}=\frac{\lambda}{2}+\rho^{*} \frac{\lambda}{2}=\frac{\lambda}{2}\left(1+\rho^{*}\right) .
$$

Similarly, when a server is busy, an incoming call will be diverted to the other server given that she is free. Therefore, the arrival rate for a busy server is

$$
\lambda_{2}=\frac{\lambda}{2}-\left(1-\rho^{*}\right) \frac{\lambda}{2}=\rho^{*} \frac{\lambda}{2} .
$$

$$
W_{P}(x)=\left\{\begin{array}{cl}
\frac{\lambda^{P} S^{2}(x)(S(x)) P-1}{(2(P-1) !)[P-\lambda S(x)] \sum_{i=0}^{P-1}(P-i)(\lambda S(x))^{i} / i !}, & \text { for } \lambda S(x)<P \\
+\infty, & \text { otherwise, }
\end{array}\right\}
$$

$$
W_{P}(x)=\left\{\frac{\lambda^{P} S^{2}(x)(K)^{P-1}}{(2(P-1) !)[P-\lambda K] \sum_{i=0}^{P-1}(P-i)(\lambda K)^{i} / i !}, \quad \text { for } \lambda K<P\right\}
$$


The average arrival rate for each queue is: $\left(1-\rho^{*}\right) \lambda_{1}+\rho^{*} \lambda_{2}=$ $(\lambda / 2)$. Since compared to the case with $P=1$, more calls find a server free, we propose the following modification in (7):

$$
\rho^{*}=\left(1-\rho^{*}\right) \lambda_{1} S(x)+\rho^{*} \lambda_{2} K
$$

which yields

$$
\rho^{*}=\frac{1-\sqrt{1-\lambda^{2} S(x)(K-S(x))}}{\lambda(K-S(x))} .
$$

It can be verified that $0 \leq \rho^{*}<1$ for $(\lambda / 2) K<1$. Also, when $\lambda$ is small, $\rho^{*} \simeq(\lambda / 2) S(x)$ as before.

Expression (27) is also valid when $P>2$. In this case, when a given server is busy, she will receive calls only if all the remaining servers are also busy. Therefore, in the case of $P$ servers, we can write

$$
\lambda_{2}=\left(\rho^{*}\right)^{P-1}\left(\frac{\lambda}{P}\right) .
$$

Since $\left(1-\rho^{*}\right) \lambda_{1}+\rho^{*} \lambda_{2}=(\lambda / P)$, we have $\lambda_{1}=\left(1+\rho^{*}+\left(\rho^{*}\right)^{2}+\right.$ $\left.\cdots+\left(\rho^{*}\right)^{P-1}\right)(\lambda / P)$. Substituting in (27), we get

$$
\rho^{*}=\left[\left(1-\left(\rho^{*}\right)^{P}\right) S(x)+\left(\rho^{*}\right)^{P} K\right] \frac{\lambda}{P} .
$$

This equation can be used to calculate $\rho^{*}$. Further, it can be written as

$$
\left(\rho^{*}\right)^{P}=\lambda_{2}\left[\left(1-\left(\rho^{*}\right)^{P}\right) S(x)+\left(\rho^{*}\right)^{P} K\right] .
$$

From (24) and (31), we see that $\rho=\left(\rho^{*}\right)^{P}$, as it should be since $\rho$ is the probability that a call finds all the $P$ servers busy. Also, the average time until completion of the service from the moment the server starts to move toward a call is

$$
\begin{aligned}
\frac{\left(1-\rho^{*}\right) \lambda_{1} S(x)+\rho^{*} \lambda_{2} K}{\left(1-\rho^{*}\right) \lambda_{1}+\rho^{*} \lambda_{2}} & =\left(1-\left(\rho^{*}\right)^{P}\right) S(x)+\left(\rho^{*}\right)^{P} K \\
& =(1-\rho) S(x)+\rho K \\
& =\frac{\rho}{\lambda_{2}}
\end{aligned}
$$

Thus, the average response time is

$$
T(x)=W_{P}(x)+\frac{\rho}{\lambda_{2}}-\sum_{j} h_{j} R_{j} .
$$

\section{Calculating $S(x)$ and $S^{2}(x)$ for the Multiserver Case}

To calculate $S(x)$ and $S^{2}(x)$, we make the following approximations:

$$
S(x)=Q(x)+\sum_{j} h_{j} d(x, j)+\sum_{j} h_{j} R_{j}
$$

and

$$
\begin{aligned}
& \sum_{j} h_{j} L_{j 1}^{2} \\
& =\sum_{j} h_{j}\left[E\left[\tilde{d}(j, x, r)^{2}\right]+d(x, j)^{2}+R_{j}^{2}\right. \\
& \left.\quad+2 R_{j} Q(x)+2 R_{j} d(x, j)+2 d(x, j) Q(x)\right]_{\lambda_{1}}
\end{aligned}
$$

where the subscript $\lambda_{1}$ implies that $Q(x)$ and $E\left[\tilde{d}(j, x, r)^{2}\right]$ in (33) and (34) must be evaluated at the arrival rate of $\lambda_{1}$.

\section{Trajectory of Optimal Location}

When $\lambda$ starts increasing, just as in the single server case, the optimal location moves away from the modified median. We can show that for small $\lambda$ values, the optimal location for the multiserver case (with arrival rate $P \lambda^{0}$ ) should be closer to the modified median than for an equivalent single server case (with arrival rate $\lambda^{0}$ ).

To understand the movement of the optimal location in a multiserver network, we approximate the behavior of a $P$-server network by considering $P$ equivalent $M / G / 1$ queues operating side by side and cooperating. Each queue behaves like a modified $M / G / 1$ queue where the call initiating a busy period has arrival rate $\lambda_{1}$ and subsequent calls in a busy period have arrival rate $\lambda_{2}$ as discussed earlier. Since calls that initiate a busy period do not incur queueing delay, it follows that, for each queue, the average queueing delay for calls that incur a delay will be the same as in Odoni's formulation [9]. Hence, we get as an approximation, the average queueing delay for the $P$ server network

$$
\begin{aligned}
& W_{q}=\left[\frac{\lambda_{2}}{1-\lambda_{2}(K-S(x))}\right] \\
& \times\left[\frac{\left(1-\lambda_{2} K\right) \sum_{j} h_{j} L_{j 1}^{2}+\lambda_{2} S(x) \sum_{j} h_{j} L_{j 2}^{2}}{2\left(1-\lambda_{2} K\right)}\right]
\end{aligned}
$$

which can be written as

$$
W_{q}=\frac{\lambda_{2} S^{2}(x)}{2\left(1-\lambda_{2} K\right)}, \quad 1-\lambda_{2} K>0 .
$$

Now, the expected response time [from (32)]

$$
T(x)=W_{q}+\frac{\rho}{\lambda_{2}}-\sum_{j} h_{j} R_{j} .
$$

From our numerical experience, the model gives a reasonably good approximation of the average response time for a multiserver network except when the server utilization becomes high.

Small $\lambda^{\prime} s$ : For a single server network with arrival rate $\lambda^{0}$, given $\lambda^{0} K, \lambda^{0} S(x) \ll 1$, from (19), we have

$$
T_{1}(x) \simeq \frac{1}{2} \lambda^{0} \sum_{j} h_{j} L_{j 1}^{2}+S(x)-\sum_{j} h_{j} R_{j}
$$

and for a $P$-server network with arrival rate $P \lambda^{0}$, given $\lambda_{2} K, \lambda_{2} S(x) \ll 1$, from (35), we have

$$
T_{2}(x) \simeq \frac{1}{2} \lambda_{2} \sum_{j} h_{j} L_{j 1}^{2}+S(x)-\sum_{j} h_{j} R_{j} .
$$

However, from (29), $\lambda_{2}=\left(\rho^{*}\right)^{P-1} \lambda^{0}$ and, for small $\lambda^{0}$, from (30) $\rho^{*} \simeq \lambda^{0} S(x)$. This gives

$$
T_{2}(x) \simeq\left(\frac{1}{2}\left(\lambda^{0}\right)^{P} \sum_{j} h_{j} L_{j 1}^{2}\right)(S(x))^{P-1}+S(x)-\sum_{j} h_{j} R_{j} .
$$

In (38) and (39), $S(x)$ and $\sum_{j} h_{j} L_{j 1}^{2}$ will have comparable values for both of the cases since these expressions get evaluated at an arrival rate of $\lambda^{0}$ since $\rho^{*}$ is small. It follows that $S(x)$ plays an increasingly dominant role in estimating response times for small $\lambda$ values when the number of servers increases. Therefore, if the optimal location of the service center for the single server case with arrival rate $\lambda^{0}$ is known (which we know will be somewhere on the arc of the network joining the modified median with the point with the least value of the second moment of the service time), it follows that the optimal location of the service center for a $P$ server case with arrival rate $P \lambda^{0}$ can be no farther 
from the modified median. It will lie on the arc of the network joining the modified median with the optimal location. The more the number of servers, the more will be the movement of the optimal location toward the modified median.

Intermediate $\lambda^{\prime} s$ : For small $\lambda^{\prime} s, S(x)$ will be smaller than $K$ for the optimal location which is close to the modified median. As $\lambda$ increases, $S(x)$ increases and for some $\lambda$ value, $S(x) \simeq K$. In this case, we can simplify the $W_{q}$ expressions (19) and (35). Then, for the one server case

$$
W_{q} \simeq \frac{\lambda^{2}}{2}\left(\frac{S(x)}{(1-\lambda K)}\right) \sum_{j} h_{j} L_{j 2}^{2}+\frac{\lambda}{2} \sum_{j} h_{j} L_{j 1}^{2}
$$

and for the $P$-server case

$$
W_{q} \simeq \frac{\lambda_{2}^{2}}{2}\left(\frac{S(x)}{\left(1-\lambda_{2} K\right)}\right) \sum_{j} h_{j} L_{j 2}^{2}+\frac{\lambda_{2}}{2} \sum_{j} h_{j} L_{j 1}^{2} .
$$

Since $\lambda_{2}=\left(\rho^{*}\right)^{P-1} \lambda^{0}$ and $\rho^{*}$ is a monotonically increasing function of $S(x)$, the second expression is more sensitive to a change in the $S(x)$ value. Therefore, optimal location should lie closer to the modified median in the $P$-server case as compared to the one server case.

Large $\lambda^{\prime} s$ : For large $\lambda^{\prime} s$, (35) generates large errors. However, from (22), we can see that when $\lambda K$ approaches $P, W_{P}(x)$ explodes. Therefore, the optimal location is that minimizes $W_{P}(x)$. The only variable term in $W_{P}(x)$ is $S^{2}(x)$. Also, since for large $\lambda^{\prime} s, \sum_{j} h_{j} L_{j 1}^{2}>$ $\sum_{j} h_{j} L_{j 2}^{2}, W_{P}(x)$ is not necessarily minimized at the modified median. Therefore, according to the model using Nozaki and Ross approximation, the optimal location for multiple server networks may not return to the modified median when $\lambda$ becomes high. This behavior is similar to that observed in the one server case. The following example demonstrates the trajectory of the optimal location in one particular case.

Example 2: Refer to Example 1 where now $l_{12}=1, l_{13}=2, l_{14}=$ $6, l_{15}=6, l_{23}=3, l_{24}=5, l_{25}=7, l_{34}=6, l_{35}=4, l_{45}=2 ; h_{1}=$ $h_{3}=h_{5}=0.10, h_{2}=h_{4}=0.35 ;$ server's travel speed $=1, R_{i}=1$ (constant) for $i=1, \ldots, 5$ and $P=2$ located at Node 2 .

Fig. 1 gives the comparison of optimal locations in the two server case (for $\lambda=\lambda^{0}$ ) with that in an equivalent one server case (i.e., for $\left.\lambda=\left(\lambda^{0}\right) /(2)\right)$.

We can see that the optimal location for two server case lies between the optimal location and the modified median for the equivalent one server case except at high $\lambda$ values.

\section{Multiserver Multifacility LOCATION PROBlem FOR EMERGENCY FACILITIES}

This section is a generalization of the approximate model discussed in Section III to the case of emergency networks with multiple service centers. The objective is to estimate the average response time for a network of $N$ demand nodes with $U$ service centers and $P$ mobile servers assigned to them. The number of servers in each location is given. Now, we can decide where to locate the service centers so as to minimize the overall average response time for the whole network.

Using the Approximate Hypercube Model: The approach developed is based on the technique that we presented in Section III together with the approach developed by Larson in [2] for the case when the servers return to their station following a service. In [2], the probabilities of the servers being busy are used to determine $h_{k}^{i}$, the modified fraction of calls that dispatch Server $i$ to Node $k$ given that she is free when a call arrives.

To illustrate our approach, we make use of an example. Fig. 2 depicts a 10-node network with three servers located at nodes 2, 4, and 9, which we call Servers 2, 4, and 9, respectively. Arc lengths are shown next to the links. Further, the dispatch matrix, based on the proximity of calls

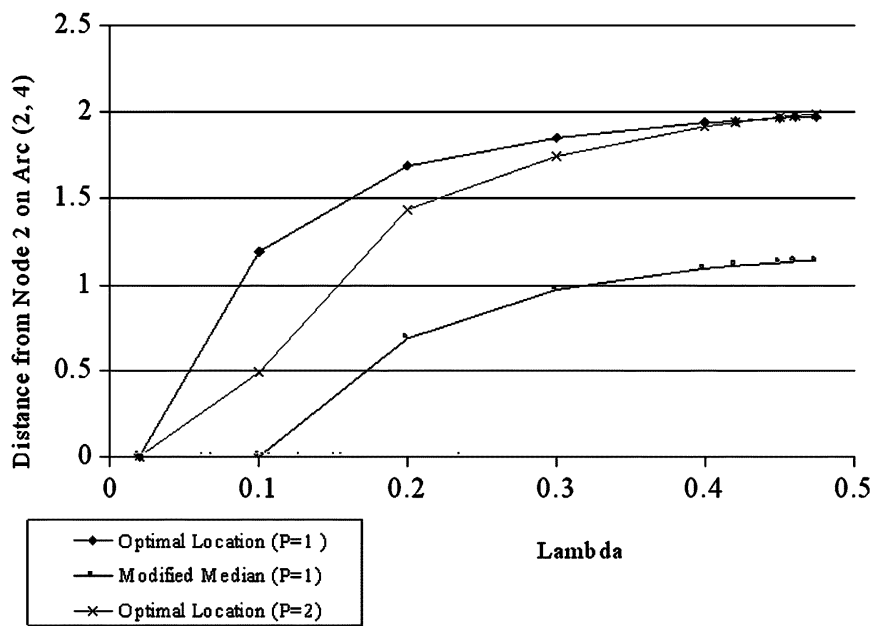

Fig. 1. Comparison of optimal locations.

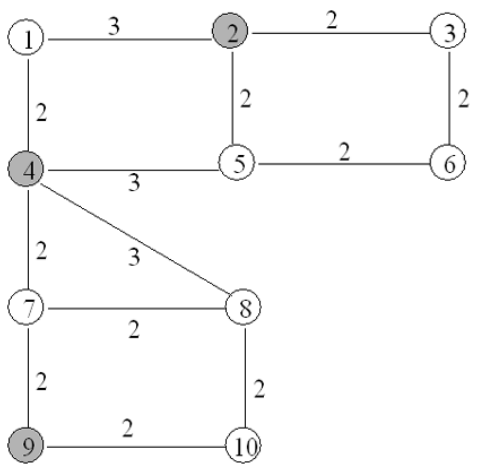

Fig. 2. Ten-node network.

TABLE II DISPATCH MATRIX FOR THE EXAMPLE

\begin{tabular}{l|r|r|r}
\hline Preference & Server 2 & Server 4 & Server 9 \\
\hline First & $2,3,5,6$ & $1,4,8$ & $7,9,10$ \\
\hline Second & 1,4 & $2,3,5,6,7,9,10$ & 8 \\
\hline Third & $7,8,9,10$ & & $1,2,3,4,5,6$ \\
\hline
\end{tabular}

to servers, is shown in Table II (e.g., if a call arrives at Node 1, Server 4 , if available, is the preferred server to be dispatched, Server 2 is the next preferred server and so on.)

Let $\rho_{2}^{*}, \rho_{4}^{*}$, and $\rho_{9}^{*}$ be, respectively, the probability of finding Servers 2,4 , and 9 busy. Assuming $\rho_{2}^{*}, \rho_{4}^{*}$, and $\rho_{9}^{*}$ are independent (a correction owing to the fact that the events of Servers 2, 4, and 9 being busy are not independent can be easily incorporated in the same way as in [2]), the effective arrival rate for Server 2 from the calls at Node $1,\left[\lambda h_{1}\right]_{2}$, is

$$
\begin{aligned}
{\left[\lambda h_{1}\right]_{2} } & =\left(1-\rho_{4}^{*}\right) 0+\rho_{4}^{*}\left(1-\rho_{2}^{*}\right) \lambda h_{1}+\rho_{4}^{*} \rho_{2}^{*} \rho_{9}^{*}\left(\frac{1}{3} \lambda h_{1}\right) \\
& =\lambda h_{1}\left(\rho_{4}^{*}-\rho_{2}^{*} \rho_{4}^{*}+\frac{1}{3} \rho_{2}^{*} \rho_{4}^{*} \rho_{9}^{*}\right) .
\end{aligned}
$$

Similarly, the effective arrival rates from Node 1 for Servers 4 and 9 , respectively, are

$$
\begin{aligned}
& {\left[\lambda h_{1}\right]_{4}=\lambda h_{1}\left(1-\rho_{4}^{*}+\frac{1}{3} \rho_{2}^{*} \rho_{4}^{*} \rho_{9}^{*}\right)} \\
& {\left[\lambda h_{1}\right]_{9}=\lambda h_{1}\left(\rho_{2}^{*} \rho_{4}^{*}-\rho_{2}^{*} \rho_{4}^{*} \rho_{9}^{*}+\frac{1}{3} \rho_{2}^{*} \rho_{4}^{*} \rho_{9}^{*}\right) .}
\end{aligned}
$$


Similarly, we can write the effective arrival rates for Servers 2,4 , and 9 for all the other nodes.

We can separate the arrival rate for Server 2 from Node 1 into two categories

$$
\left[\lambda h_{1}\right]_{2 \text { free }}=\rho_{4}^{*}\left(1-\rho_{2}^{*}\right) \lambda h_{1} \quad\left[\lambda h_{1}\right]_{2 \text { busy }}=\rho_{2}^{*} \rho_{4}^{*} \rho_{9}^{*}\left(\frac{1}{3} \lambda h_{1}\right) .
$$

Notice that $\forall i:\left[\lambda h_{i}\right]_{2 \text { busy }}=\rho_{2}^{*} \rho_{4}^{*} \rho_{9}^{*}\left((1 / 3) \lambda h_{i}\right)$. Now, we can calculate the effective arrival rate for Server 2 , both when free, $\lambda_{2 \text { free, }}$, and when busy, $\lambda_{2 \text { busy }}$ and also the corresponding effective fractions of demand at various nodes when Server 2 is free, $\left[h_{i}\right]_{2 \text { free }}$ and when busy, $\left[h_{i}\right]_{2 \text { busy }}$

$$
\begin{aligned}
\lambda_{2 \text { free }} & =\sum_{i=1}^{10}\left[\lambda h_{i}\right]_{2 \text { free }} \\
\lambda_{2 \text { busy }} & =\sum_{i=1}^{10}\left[\lambda h_{i}\right]_{2 \text { busy }}=\rho_{2}^{*} \rho_{4}^{*} \rho_{9}^{*}\left(\frac{1}{3} \lambda\right)
\end{aligned}
$$

and

$$
\begin{aligned}
{\left[h_{i}\right]_{2 \text { free }} } & =\frac{\left[\lambda h_{i}\right]_{2 \text { free }}}{\lambda_{2 \text { free }}} \\
{\left[h_{i}\right]_{2 \text { busy }} } & =\frac{\left[\lambda h_{i}\right]_{2 \text { busy }}}{\lambda_{2 \text { busy }}}=h_{i} .
\end{aligned}
$$

Arrival Rate Calibration: Let $\lambda_{2}=\sum_{i=1}^{10}\left[\lambda h_{i}\right]_{2}$ be the overall arrival rate for Server 2 . Then, from above, we have $\lambda_{2 \text { free }}+\lambda_{2 \text { busy }}=\lambda_{2}$.

However, since Server 2 is free for $\left(1-\rho_{2}^{*}\right)$ fraction of time and busy for $\rho_{2}^{*}$, the effective arrival rates for free and busy periods must satisfy $\lambda_{2 \text { free }}^{c}\left(1-\rho_{2}^{*}\right)+\lambda_{2 \text { busy }}^{c} \rho_{2}^{*}=\lambda_{2}$ where $\lambda_{2 \text { free }}^{c}$ and $\lambda_{2 \text { busy }}^{c}$ are the calibrated effective arrival rates for free and busy periods of Server 2: $\lambda_{2 \text { free }}^{c}=\left(\lambda_{2 \text { free }}\right) /\left(1-\rho_{2}^{*}\right)$ and $\lambda_{2 \text { busy }}^{c}=\left(\lambda_{2 \text { busy }}\right) /\left(\rho_{2}^{*}\right)$. Similarly, the calibrated effective arrival rates for free and busy periods of Servers 4 and 9 can be calculated.

Solution Procedure: For each server, using (41)-(43), calculate $\lambda_{\text {free }}^{c}$ and $\left[h_{i}\right]_{\text {free }}$, the overall arrival rate and fraction of demands at each node when the server is free, and $\lambda_{\text {busy }}^{c}$ and $\left[h_{i}\right]_{\text {busy }}$, which represent the same quantities for the case when the server is busy. These are sufficient to calculate the first and second moments of the service time $S(x)$ and $K$, defined in Section III, for each of the servers independently. From the multiserver model with one service station (27), we know that

$$
\begin{aligned}
& \rho_{2}^{*}=\left(1-\rho_{2}^{*}\right) \lambda_{2 \text { free }}^{c} S\left(x_{2}\right)+\rho_{2}^{*} \lambda_{2 \mathrm{busy}}^{c} K_{2} \\
& \rho_{4}^{*}=\left(1-\rho_{4}^{*}\right) \lambda_{4 \text { free }}^{c} S\left(x_{4}\right)+\rho_{4}^{*} \lambda_{4 \mathrm{busy}}^{c} K_{4} \\
& \rho_{9}^{*}=\left(1-\rho_{9}^{*}\right) \lambda_{9 \text { free }}^{c} S\left(x_{9}\right)+\rho_{9}^{*} \lambda_{9 \mathrm{busy}}^{c} K_{9}
\end{aligned}
$$

where $S\left(x_{k}\right)$ and $K_{k}$ correspond to $S(x)$ and $K$ values for Server $k=2,4,9$. Therefore, knowing $\lambda_{k \text { free }}^{c} S\left(x_{k}\right), \lambda_{k \text { busy }}^{c}$, and $K_{k}$ for Server $k$, we can get $\rho_{k}^{*}, k=2,4,9$.

Following [2], the solution procedure consists of starting with $\rho_{2}^{*}=$ $\rho_{4}^{*}=\rho_{9}^{*} \simeq 0$ to obtain $\lambda_{k \text { free }}^{c},\left[h_{i}\right]_{k \text { free }}, \lambda_{k \text { busy }}^{c}$, and $\left[h_{i}\right]_{k \text { busy }}$ for each of the three servers. Then, we calculate $S\left(x_{k}\right)$ and $K_{k}$ for Server $k$ and use (44) to calculate new values of $\rho_{k}^{*}, k=2,4,9$. With the new $\rho_{k}^{*}, k=2,4,9$ we calculate new values for $\lambda_{k \text { free }}^{c}$ and $\lambda_{k \text { busy }}^{c}$ for $k=2,4,9$, and so on until $\rho_{k}^{*}, k=2,4,9$ values converge.

Calculating $W_{q}$ : With all the parameters now known, we can approximately calculate $W_{q}$. We know that a queue will form only in the case where all the three servers are busy. However, in this case, the overall arrival rate is simply $\lambda$ and the servers go from node to node and do not go back to the service center. Therefore, we can regard the servers as homogeneous and use the expression in (22) as an approximation. Here, $K$ is the same for all the servers but $S^{2}\left(x_{k}\right)$ is different and we propose to substitute $S^{2}(x)=\left(\sum_{k=1}^{P} S^{2}\left(x_{k}\right) / P\right.$ in (22). $S^{2}\left(x_{k}\right)$ can be computed using (23), (24), (33), and (34).

1) Generalizing the Procedure: Let $\rho_{k}^{*}$ be the probability that Server $\mathrm{k}$ is busy. Using the terminology in [2], let $n_{i l}$ be the identification number of the $l$ th preferred server for a call originating at Node $i$. Let Server $k$ be the $j$ th preferred server for a call originating at Node $i$, i.e., $n_{i j}=k$. Then, the arrival rate for Server $k$ from Node $i$ is

$$
\left[\lambda h_{i}\right]_{k}=\left(\prod_{l=1}^{j-1} \rho_{n_{i l}}^{*}\right)\left(1-\rho_{k}^{*}\right) \lambda h_{i}+\frac{1}{P}\left(\prod_{l=1}^{P} \rho_{l}^{*}\right) \lambda h_{i} .
$$

This expression can be separated into

and

$$
\left[\lambda h_{i}\right]_{k \text { free }}=\left(\prod_{l=1}^{j-1} \rho_{n_{i l}}^{*}\right)\left(1-\rho_{k}^{*}\right) \lambda h_{i}
$$

$$
\left[\lambda h_{i}\right]_{k \text { busy }}=\frac{1}{P}\left(\prod_{l=1}^{P} \rho_{l}^{*}\right) \lambda h_{i} .
$$

The remaining expressions are easily generalized. As before, we can calculate the effective arrival rate and the corresponding fractions of demand at various nodes for Server $k$, when free and busy

$$
\begin{aligned}
\lambda_{k \text { free }} & =\sum_{i=1}^{N}\left[\lambda h_{i}\right]_{k \text { free }}, \quad \lambda_{k \text { busy }}=\sum_{i=1}^{N}\left[\lambda h_{i}\right]_{k \text { busy }} \\
& =\left(\prod_{l=1}^{P} \rho_{l}^{*}\right)\left(\frac{\lambda}{P}\right)
\end{aligned}
$$

and

$$
\left[h_{i}\right]_{k \text { free }}=\frac{\left[\lambda h_{i}\right]_{k \text { free }}}{\lambda_{k \text { free }}}, \quad\left[h_{i}\right]_{k \text { busy }}=h_{i} .
$$

Let $\lambda_{k}=\sum_{i=1}^{N}\left[\lambda h_{i}\right]_{k}$ be the overall arrival rate for Server $k$. Then, $\lambda_{k \text { free }}+\lambda_{k \text { busy }}=\lambda_{k}$ and thus $\lambda_{k \text { free }}^{c}\left(1-\rho_{k}^{*}\right)+\lambda_{k \text { busy }}^{c} \rho_{k}^{*}=\lambda_{k}$.

The calibrated effective arrival rates for the free and busy periods of Server $k$ are: $\lambda_{k \text { free }}^{c}=\left(\lambda_{k \text { free }}\right) /\left(1-\rho_{k}^{*}\right)$ and $\lambda_{k \text { busy }}^{c}=$ $\left(\lambda_{k \text { busy }}\right) /\left(\rho_{k}^{*}\right)$. Finally, $\rho_{k}^{*}=\left(1-\rho_{k}^{*}\right) \lambda_{k \text { free }}^{c} S\left(x_{k}\right)+\rho_{k}^{*} \lambda_{k \text { busy }}^{c} K_{k}$.

\section{A. Evaluating Performance of the Approximate Model}

\section{Comparing Average Response Times}

Example 3: Fig. 3 depicts the average response times for the network shown in Fig. 2 obtained from the model and those from simulation for different $\lambda$ values. Here, $P=U=3$. We use $h_{1}=0.2, h_{2}=$ $0.05, h_{3}=0, h_{4}=0.2, h_{5}=0.1, h_{6}=0.1, h_{7}=0.15, h_{8}=$ $0, h_{9}=0.1, h_{10}=0.1$, and $R_{i}=1, i=1$ to 10 , the on-site service time is assumed to be a constant.

As can be seen from the figure, the approximation works quite well in terms of the average response time.

A Heuristic Procedure to Find 'The Optimal Set of Server Locations': We assume that we have a finite set of potential locations. For all the examples that follow, we limit our search to the set of nodes (non-nodal locations can be incorporated as well).

We use a heuristic algorithm similar to the one presented in [11].

1) Start with an initial set of locations.

2) Assume $\rho_{k}^{*} \simeq 0, k=1, \ldots, P$. Calculate $\lambda_{\text {free }}^{c},\left[h_{i}\right]_{\text {free }}, \lambda_{\text {busy }}^{c}$, and $\left[h_{i}\right]_{\text {busy }}$ for each of the $P$ servers.

3) Calculate $S(x)$ and $K$ for each server. Use (44) to obtain new values of $\rho_{k}^{*}$. With the new $\rho_{k}^{*}$, calculate new values for $\lambda_{k}^{c}$ free and $\lambda_{k \text { busy }}^{c}$ etc., and continue until the $\rho_{k}^{*}$ values converge.

4) With $\lambda_{\text {free }}^{c},\left[h_{i}\right]_{\text {free }}, \lambda_{\text {busy }}^{c}$, and $\left[h_{i}\right]_{\text {busy }}$ found for each server, determine the optimal location for each server independently treating the problem as a single server network problem. 


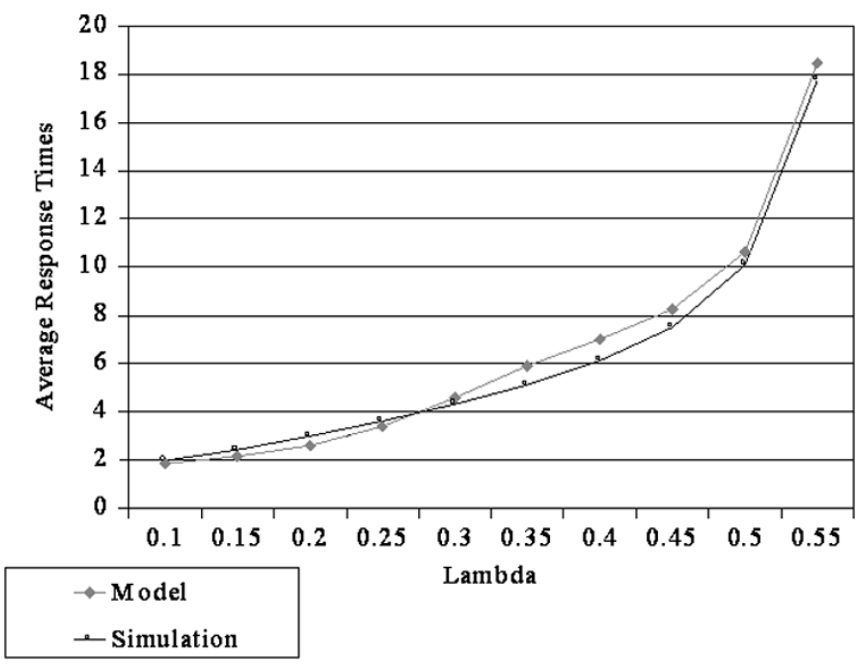

Fig. 3. Comparison of average response times for Example 3.

TABLE III

OPtimal SET OF LOCATIONS AS A FUNCTION OF $\lambda$

\begin{tabular}{l|l|l|c|c}
\hline$\lambda$ & Set of & \multicolumn{2}{|l|}{ Average Response Time } & \multirow{2}{*}{ \% Error } \\
\cline { 3 - 4 } & Optimal Locations & Model & Simulation & \\
\hline \multirow{2}{*}{.001} & Starting $\{1,3,8\}$ & 4.651 & & \\
& Final $\{5,4,9\}$ & 1.202 & 1.198 & 0.3 \\
.1 & $\{5,4,9\}$ & 1.526 & 1.65 & -7.5 \\
.2 & $\{5,4,9\}$ & 2.18 & 2.54 & -14.2 \\
& Starting $\{5,4,9\}$ & 2.81 & 3.19 & -11.9 \\
.25 & Final $\{5,4,7\}$ & 2.76 & 2.97 & -7.1 \\
.3 & $\{5,4,7\}$ & 3.52 & 3.57 & -1.4 \\
& Starting $\{5,4,7\}$ & 4.62 & 4.3 & 7.4 \\
.35 & Final $\{4,4,7\}$ & 4.27 & 4.09 & 3.9 \\
.4 & $\{4,4,7\}$ & 5.26 & 4.95 & 6.3 \\
\multirow{2}{*}{.45} & Starting $\{4,4,7\}$ & 6.71 & 6.2 & 8.2 \\
& Final $\{4,4,4\}$ & 6.53 & 6.15 & 6.2 \\
.5 & $\{4,4,4\}$ & 9.01 & 8.75 & 3.0 \\
.55 & $\{4,4,4\}$ & 16.96 & 16.88 & 0.5 \\
\hline
\end{tabular}

5) With this obtained set of optimal locations, go back to Step 2 above and continue this procedure. Stop when the set of optimal locations does not change in two consecutive iterations.

We note that in all our computational experience, convergence has been obtained.

Example 4: We consider again Example 3. Here, $P=3$ but $U$, the number of service centers, is determined by the model. In Table III, we show the set of optimal locations given by the model for various $\lambda$ values. The set of locations $\{1,3,8\}$ was chosen arbitrarily to start the procedure with $\lambda=0.001$. The model determined the 'optimal' set to be $\{5,4,9\}$. This set remained optimal for $\lambda=0.1$ and 0.2 but for $\lambda=0.25$, starting with $\{5,4,9\}$, the optimal set changed to $\{5,4,7\}$. Finally, for high $\lambda$ values, $\{4,4,4\}$ became the optimal set. Table III also shows the corresponding average response times given by the model and simulation.

Trajectory of Optimal Location: According to this heuristic, the "optimal" set of locations is seen to move from the 3-modified median, when $\lambda$ is close to zero, toward the 1-modified median as $\lambda$ becomes high. This is because for small $\lambda^{\prime} s$, the servers are mostly free and therefore service demands arising mainly from their preferred nodes.

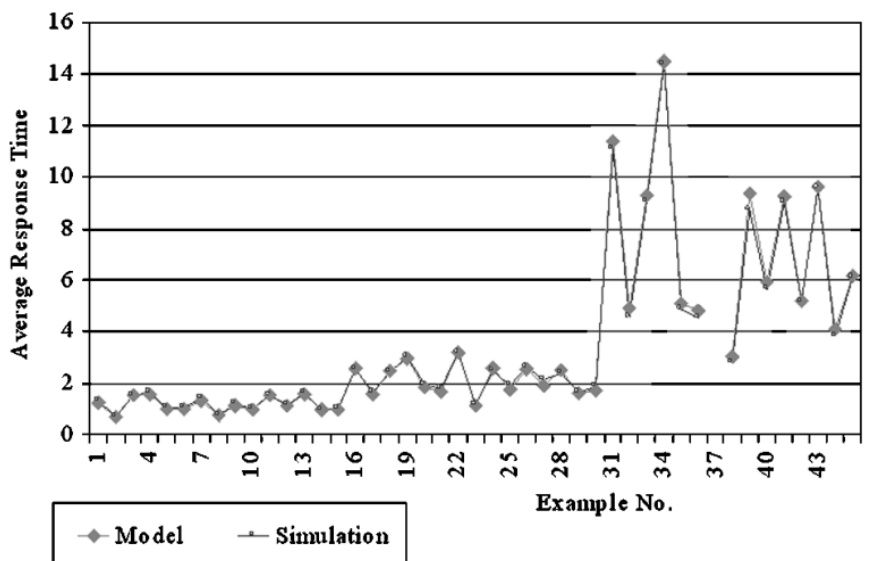

Fig. 4. Comparison of the average response times for the 45 examples.

When the system starts becoming congested, servers are busy most of the time and therefore demands from various nodes get distributed among servers more and more uniformly. Hence, the movement from a P-median toward a 1-median which is also supported by simulation results.

To further test the performance of the approximate model, we use the following set of 45 examples.

We compare the average response time in the case of 15 randomly generated networks with eight nodes, $P=3$ and the number of service centers, $U$, as determined by the model. The performance of these fifteen networks was compared with simulation at three different $\lambda$ values, $\lambda \in\{0.1,0.3,0.6\}$. The coordinates of the nodes were generated randomly. The values $R_{i} \in[1,2]$ were generated from a uniform random distribution. Further, $h_{i}$ were calculated indirectly by (uniformly) randomly generating $(1) /\left(\lambda_{i}^{\prime}\right) \in[100,1000]$ so that $h_{i}=$ $\left(\lambda_{i}^{\prime}\right) /\left(\sum \lambda_{i}^{\prime}\right)$. Servers were assumed to travel with unit speed and service centers are located at the optimal set of locations as given by our algorithm for the corresponding $\lambda$ value.

Fig. 4 shows the comparison of the average response times obtained from the model and from simulation for the 45 examples generated above. For Examples 1-15, $\lambda=0.1$. In this case, the server utilization varies from $8 \%$ to $10 \%$. Thus the system is relatively free. The mean absolute percentage error (MAPE) is found to be $3.5 \%$. For Examples $16-30, \lambda=0.3$. Here, the average server utilization varies from $30 \%$ to $40 \%$ and MAPE is $4.5 \%$. Finally, Examples $31-45$ use $\lambda=0.6$. The average server utilization varies from $75 \%$ to $90 \%$ and the MAPE is $4.1 \%$. Example 37 is not shown as simulation became unreliable on account of long queues.

Based on this comparison, the approximate model gives reasonably good estimates of the average response time.

\section{ACKNOWLEDGMENT}

The authors would like to thank the referees for their useful suggestions.

\section{REFERENCES}

[1] R. C. Larson, "A hypercube queueing model for facility location and redistricting in urban emergency services," Comput. Oper. Res., vol. 1, pp. 67-95, 1974.

[2] - "Approximating the performance of urban emergency service systems," Oper. Res., vol. 23, no. 5, pp. 845-868, 1975. 
[3] J. P. Jarvis, "A location model for spatially distributed queueing systems," in Proc. IEEE Int. Conf. Syst., Man, Cybern., Nov. 1976, pp. $32-35$.

[4] O. Berman, R. C. Larson, and S. S. Chiu, "Optimal server location on a network operating as an $M / G / 1$ queue," Oper. Res., vol. 33, no. 4, pp. 746-770, July-Aug. 1985.

[5] - "Locating a mobile server queueing facility on a tree network," Manage. Sci., vol. 31, no. 6, pp. 764-772, 1985.

[6] S. S. Chiu, "A dominance theorem for the stochastic queue median problem," Oper. Res., vol. 34, pp. 942-944, 1986.

[7] - "Optimal $M / G / 1$ server location on a tree network with continuous link demands," Comput. Oper. Res., vol. 13, pp. 653-669, 1986.

[8] R. Batta, "The stochastic queue median over a finite discrete set," Oper. Res., vol. 37, no. 4, pp. 648-652, 1989.

[9] — "Single server queueing-location models with rejection," Trans. Sci., vol. 22, no. 3, pp. 209-216, 1988.

[10] R. Batta and O. Berman, "A location model for a facility operating as an $M / G / k$ queue," Networks, vol. 19, pp. 717-728, 1989.
[11] O. Berman, R. C. Larson, and C. Parkan, "The stochastic queue $p$-median problem," Trans. Sci., vol. 21, no. 3, pp. 207-216, 1987.

[12] M. Jamil, R. Batta, and D. M. Malon, "The travelling repairperson home base location problem," Trans. Sci., vol. 28, no. 2, pp. 150-161, 1994.

[13] P. B. Mirchandani and R. L. Francis, Eds., "Discrete location theory, chapter on location of mobile units in a stochastic environment," in Discrete Mathematics and Optimization. ser. Interscience Series. New York: Wiley, 1990, pp. 503-549. by O. Berman, S. S. Chiu, R. C. Larson, A. R. Odoni, and R. Batta.

[14] A. R. Odoni, "An analytical investigation of air traffic in the vicinity of terminal areas," Ph.D. dissertation, Aeronautics Dept., Mass. Inst. Technol., Cambridge, 1969.

[15] O. Berman and D. Krass, "Facility location problems with stochastic demands and congestion," in Location Analysis: Applications and Theory, Z. Drezner and H. W. Hamacher, Eds. New York: Springer, 2002, ch. 11, pp. 329-371. 